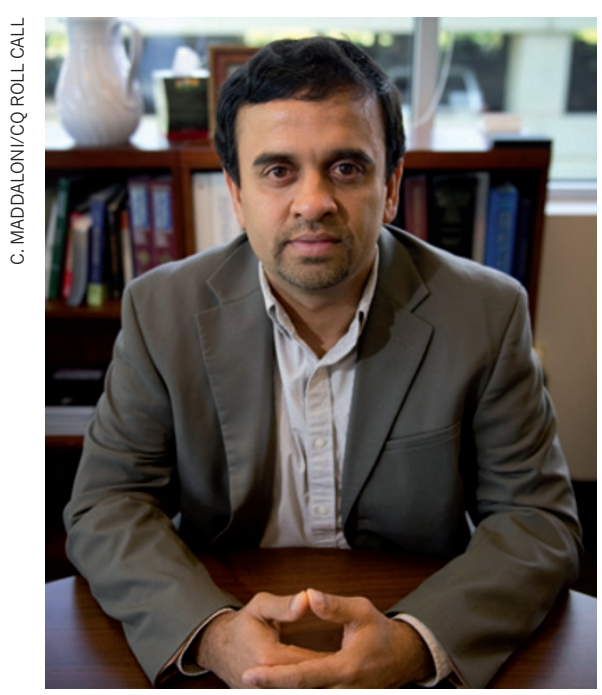

Rajesh Ranganathan says that getting industrial experience in drug discovery is key for academics.

that would not normally be pursued by industry (see Nature 461, 554-555; 2009).

The programmes aim to address one of the main concerns about industrial research, which was "getting more and more entangled in engineered research where everything was milestones and very short-sighted", says Klaus Müller, a medicinal chemist at Roche. To keep from "drying out", companies such as Roche recruit top scientists, promote cuttingedge science and encourage publication. At the same time, postdocs in company programmes have industry resources at their disposal, and gain experience in drug discovery

But an element of caution is needed, says Ranganathan, who worked at Novartis and set up the company's postdoctoral fellow programme before moving to the NINDS. "Make sure that such a programme is not a reward structure for internal scientists." Otherwise academic postdocs act as a "pair of hands for those people who are already successful in the company", he says, rather than as well-mentored trainees and achievers of scientific renown for themselves.

\section{A PRODIGIOUS PARTNER}

Academia-industry collaborations can be a way to both gain drug-discovery expertise and advance a project. Organic chemist Erick Carreira, at the Swiss Federal Institute of Technology in Zurich (ETHZ), followed that path when he began to collaborate with scientists at Roche. Before he came to Switzerland from the United States, his group was synthesizing new molecules and testing them through standard chemical means, such as measuring boiling and melting points or solubility. He never thought about designing drugs.

But shortly after joining the ETHZ 15 years ago, Carreira became a consultant with Roche; together, he and Roche scientists came up with a wild, "scribbled-on-a-napkin" idea. What if they introduced a new kind of chemical unit into potential drugs to help them to work better?

The idea was to hunt outside the usual 'chemical space', searching for all the possible molecules of a given class, to look for structures with properties deemed 'useful' in the drug-discovery process. Müller identified a new class of synthetic chemicals called oxetanes as an under-explored area, and Carreira began modelling ways to substitute oxetanes for common functional groups already used in drug discovery. Normally, industrial scientists would consider such an idea too "odd, too risky and unlikely to succeed”, says Müller, who is secretary of the RPF Program. But for academics such as Carreira and his team, the project was perfect.

Roche backed the idea, supporting a synthetic-chemistry student, Georg Wuitschik, to work with Carreira, Müller and others to usher oxetanes into drug discovery. The results paid off, and the scientists published what became known as the oxetane concept (G. Wuitschik et al. J. Med. Chem. 53, 3227$3246 ; 2010)$. It rapidly took hold in the drugdiscovery community, offering potentially new ways to modify drugs. "This is really a gold mine," says Müller.

Meanwhile, Carreira, in collaboration with Roche, is coaching chemistry students, postdocs and others who are interested in drug design. "The requirements of someone now coming out of graduate school in synthetic chemistry have changed," he says. "Given the current job climate, a graduate student or postdoc needs to be fully conversant not only with the methods of chemical synthesis - how to make molecules - but also how to make novel chemicals with a pivotal role in the next generation of smart drugs."

For Zylka, working with an industry veteran who had returned to academia was key. Before coming to UNC, Frye, as head of GSK's discovery medicinal-chemistry group, had steered three drugs either to market or late-stage clinical trials. His team offers the best of both worlds, Zylka believes: the creativity and freedom of an academic lab and the wisdom and experience of an industrial mentor.

Those in academia who can take advantage of such industry ties and drug-discovery resources have a shot at a grand pay-off, and a new career path - if they dare to be bold, enterprising and an "expert at one discipline but knowledgeable about the other translational disciplines", says Austin.

"The scientific ecosystem has evolved to a place where rapid advances will happen," he adds. "And basic scientists can actually make that happen without going to a company. This is absolutely revolutionary."

Trisha Gura is a science writer in Boston, Massachusetts.

\section{CITATION}

\section{Data standards urged}

The academic community needs better curation and authorship standards for data, according to a committee of the US National Academies. In For Attribution Developing Data Attribution and Citation Practices and Standards, published on 19 November, the Board on Research Data and Information (BRDI) presents views from experts in data curation across all scientific disciplines. It says that scientific data - such as measurements and images - should be made available for scrutiny online or through an archive or repository, and that researchers should be given full credit for their efforts in creating those data. The report notes that some scientific associations, including the American Geophysical Union, endorse giving the same importance to the publication of scientific data as to the publication of papers.

Data citation, the committee says, gives authors proper credit, makes them accountable and helps to make science reproducible. Although it offers no formal recommendations, the report suggests that digital object identifiers similar to those used for research papers would provide a permanent online address for data sets and allow the data to be cited formally.

"People are using other people's data more often, so they need a way to cite and attribute sources," says Paul Uhlir, the committee rapporteur and director of the BRDI. "Up to now, there has been no convention for it. That's really behind the whole rise in calls for data citation and attribution standards - it's an infrastructure issue. Those people need credit."

The report notes that questions remain regarding how to decide which data are curated, who would pay to maintain data in repositories or archives and who would peer review data and how.

Uhlir says that how often a researcher's data are cited is likely to become an important career metric. The report suggests that it could be incorporated into tenure decisions, as paper citations and journal impact factors are now.

For Attribution is the first of several expected publications on the subject. The BRDI will contribute to a report to be released next year by the international Committee on Data for Science and Technology (CODATA), part of the International Council for Science in Paris on current practices in data curation and authorship. A final report from CODATA presenting best practices and recommendations is expected by 2014 . 